\title{
Increased childhood BMI is associated with young adult serum uric acid levels: a linkage study from Japan
}

\author{
Erika Kuwahara', Yoshitaka Murakami ${ }^{2}$, Tomonori Okamura ${ }^{3}$, Hirokazu Komatsu' ${ }^{4}$ Akemi Nakazawa ${ }^{5}$, Hideo Ushiku ${ }^{6}$, \\ Fumio Maejima ${ }^{5}$, Yoshio Nishigaki ${ }^{5}$ and Yuji Nishiwaki ${ }^{1}$
}

\begin{abstract}
BACKGROUND: Growth pattern in early life is one of the most important factors affecting the pathogenesis of metabolicassociated diseases. The associations between serum uric acid (SUA) and hypertension, kidney disease, and coronary heart disease have been recognized. We investigated the association between increased BMI during childhood and adult SUA levels in Japan.
\end{abstract}

METHODS: We included 298 children with health examination data between 1981 and 2002 who had also undergone physical examinations after reaching early adulthood (approximately 27 y old). Subjects were divided into sex-specific tertiles based on the difference in their BMI (DBMI) over a 6-y period (6-12 y of age). The association between the three DBMI groups and SUA in adults was analyzed.

RESULTS: The predicted average SUA level in adults from the high DBMl group was $5.32 \mathrm{mg} / \mathrm{dl}$ after adjustment for related factors in a combined sex analysis. This was significantly higher than among the low DBMI group.

CONCLUSION: Excessive BMI increases during childhood led to young adult SUA elevation even after adjusting for several factors. Lifestyle in early life may be a strong predictor of future uric acid metabolism and the resulting disease risk.

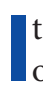
t has been well recognized that uric acid causes gout and is one of the markers for several lifestyle-related diseases. Since the 1950s, the associations between serum uric acid (SUA) and hypertension (1), kidney diseases (2), and coronary heart disease (3) have been recognized. Increased SUA level may be an independent risk factor for chronic kidney disease $(4,5)$, stroke (6), coronary heart disease (7), and cardiovascular diseases (8). Moreover, some studies have shown that SUA is a risk factor for metabolic syndrome among children and adolescents $(9,10)$. These findings suggest that prevention of SUA level elevation in early life has a potential protective effect.

The association between SUA and obesity is also well known. Overweight and obesity among children have been globally recognized as a public health concern. Recently, growth pattern during early life, not merely a single assessment of childhood body weight or BMI, is considered to be one of the most important risk factors for the pathogenesis of metabolic-associated diseases (11-17). However, to the best of our knowledge, no study has evaluated the association between early-life growth patterns and SUA levels later in life. Therefore, we investigated the association between increased BMI during childhood and subsequent SUA level during early adulthood in Japan.

\section{METHODS}

\section{Study Population and Design}

The Japanese law mandates annual physical examinations for students in all schools in Japan. We obtained anonymous secondary data from these examinations from towns A and B in Nagano Prefecture, with populations of approximately 11,000 and 5,000, respectively, in 2015. Previously, we performed a similar linkage study in town A to investigate the association between an increase in BMI during $6 \mathrm{y}$ of primary school and blood pressure in adolescents (18). Data collection was started in 1981 in town A and 1996 in town B. Town A has one and town B has two primary schools, which were attended by almost all children living in this area. The eligible study population comprised 1,711 children ( 850 boys and 861 girls) who entered primary school (aged 6 y) by 2002 . Of these, 1,621 (809 boys and 812 girls) graduated from the same school when they were $12 \mathrm{y}$ old. After the age of $18 \mathrm{y}$, 298 (18.4\%; 144 boys and 154 girls) remained in the same town or a neighboring area and received a health examination, including blood tests at the local hospital (Saku Central Hospital) at least once.

\section{Physical Examinations and Laboratory Measurements}

Regular mandatory school physical examinations included a medical interview, height, and weight each school year. Height and weight were measured by a school nurse and rounded to the nearest integer. BMI was calculated as weight in kilograms divided by the square of height in meters. To confirm the representativeness of the sample, we converted BMI to BMI SD scores (BMI-SDSs) according to the LMS method $(19,20)$. The mean \pm SD of BMI-SDSs for all first-grade students (aged 6 or 7 y) were $-0.10 \pm 0.87$. (boys, $-0.12 \pm 0.91$; girls, $-0.08 \pm 0.83$ ). The BMI-SDS for sixth-grade students (aged 11 or $12 \mathrm{y}$ ) were $-0.06 \pm 1.03$ (boys, $-0.01 \pm 1.03$; girls, $-0.11 \pm 1.04$ ). The BMISDS analysis verified that the subjects were representative of Japanese school children in terms of BMI.

DBMI was calculated for each subject and divided into sex-specific tertiles (low, moderate, and high for the first, second, and third tertiles, respectively). These tertiles were used as an indicator of physical change. Japanese primary schools include first through sixth grades;

\footnotetext{
'Department of Environmental and Occupational Health, School of Medicine, Toho University, Tokyo, Japan; ${ }^{2}$ Department of Medical Statistics, School of Medicine, Toho University, Tokyo, Japan; ${ }^{3}$ Department of Preventive Medicine and Public Health, Keio University School of Medicine, Tokyo, Japan; ${ }^{4}$ Department of Community Care, Saku Central Hospital, Nagano, Japan; ${ }^{5}$ Division of Health Care, Saku Central Hospital, Nagano, Japan; ${ }^{6}$ Department of Pediatrics, Saku Central Hospital, Nagano, Japan. Correspondence: Erika Kuwahara (erk61022@gmail.com)

Received 28 March 2016; accepted 9 August 2016; advance online publication 30 November 2016. doi:10.1038/pr.2016.213
} 
thus, the use of DBMI during this period is convenient for continuous health assessment. Guardians of children who were overweight or believed to be unhealthy in any way after the examination were advised to seek medical advice. However, no systematic interventions relating to obesity in children were implemented during the observation period.

The mandated regular physical examination for adults included blood tests, blood pressure, and a chest X-ray in addition to the aforementioned items on the school health examination. SUA and Cre were measured using the uricase-peroxidase method and creatinasesarcosine oxidase-peroxidase method, respectively (Hitachi 73650E and Hitachi 7600-300, respectively; Hitachi High-Technologies Corporation, Tokyo, Japan). Blood pressure was measured by nurses using an automated oscillometric sphygmomanometer (ES-H51T3 or ES-H55; Terumo Corporation, Tokyo, Japan).

\section{Statistical Analysis}

For comparing the tertiles of DBMI, ANOVA and post hoc tests with Bonferroni correction were used for continuous variables, and Pearson's chi-square tests were used for categorical variables. Next, we conducted a linear regression analysis to examine the association between DBMI and SUA among young adults after adjusting for other covariates. Model 1 was used for the crude analysis of the association between DBMI and SUA. Model 2 included BMI year of entrance, and area of residence during the first grade as covariates. Sex was also adjusted for in analyses that included boys and girls. Model 3 included BMI, Cre, systolic blood pressure (SBP), and age at the adult follow up, in addition to the variables in Model 2. SUA and all covariates except for sex and area of residence were treated as continuous variables. Using these regression equations, predicted SUA levels were calculated by simply substituting a value $(0,1$, or 2$)$ for the explanatory variable (low, moderate, or high DBMI, respectively) and substituting average values for the other independent variables (with the margins command in STATA).

We conducted three additional analyses. First, we only included subjects who had information on alcohol consumption at the adult examination $(n=236)$ and repeated the analysis with adjustment for alcohol consumption and the variables included in Model 3. Second, the representativeness of the sample was assessed by a comparison of baseline characteristics between the group with childhood data alone and this study population using Student's $t$-test. Third, SUA levels were classified as $<7 \mathrm{mg} / \mathrm{dl}$ and $\geq 7 \mathrm{mg} / \mathrm{dl}$, using the cutoff level for hyperuricemia that was recommended by the Japanese Society of Gout and Nucleic Acid Metabolism (21,22), and a logistic regression analysis was conducted using the same covariates as Models. Thirtythree of the 34 subjects with a high SUA level were males; therefore, this logistic regression analysis was only conducted for males.

All analyses were performed with STATA, version 12.0 (STATA, College Station, TX). All CIs were estimated at the 95\% level, and $P$ values of $<0.05$ were considered statistically significant.

\section{Ethical Considerations}

All data provided by Saku Central Hospital were anonymized and rendered unlinkable after they were given an identification number for research. Thus, the researchers could not access personal information for any of the subjects. Therefore, the requirement for informed consent was waived according to the Ethical Guidelines for Medical and Health Research Involving Human Subjects in Japan. This study was approved by Saku Central Hospital's Ethics Committee (approval number: 31 ) and the Ethics Committee of Toho University, Faculty of Medicine (approval number: 25011).

\section{RESULTS}

Table 1 shows subjects' baseline characteristics by tertile of difference in BMI (DBMI). The mean \pm SD of DBMI between the first and sixth grades was $2.9 \pm 2.4$ in boys and $2.7 \pm 1.9$ in girls ( $P=0.82$, Mann-Whitney $U$-test). Most baseline variables, except for area of residence during the first grade overall and for boys, differed among tertiles of DBMI.
Figure 1 shows the distribution of SUA after reaching adulthood. The peak totals reached $6.0-6.49 \mathrm{mg} / \mathrm{dl}$ for boys and $4.0-4.49 \mathrm{mg} / \mathrm{dl}$ for girls. Table 2 shows the characteristics at the last health examination after $18 \mathrm{y}$ of age (mean age: $26.7 \mathrm{y}$ ) by tertile of DBMI. There was a significant difference in young adult SUA between the tertiles of DBMI. Among both boys and girls, SUA differed by approximately $0.4 \mathrm{mg} / \mathrm{dl}$ between the low and high DBMI groups. BMI and weight maintained similar tendencies as that of SUA between the three groups for both sexes.

Table 3 shows the predicted SUA levels from the result of the sex-combined and sex-stratified univariate (unadjusted) regression analysis. There was no statistical interaction between sex and DBMI for SUA $(P=0.45)$. The high DBMI group had a higher adult SUA level than the low DBMI group for the combined sex analysis (Model 1, $P=0.009$ ). These associations persisted after adjusting for childhood BMI, year of entrance to the primary school, and area of residence (Model 2, $P=0.001)$. When we further adjusted for adult BMI, serum creatinine (Cre), SBP, and age (Model 3), the positive association between DBMI and SUA was attenuated but remained statistically significant $(P=0.014)$. Further adjustment by alcohol consumption did not change the results substantially. The adjusted difference for the high DBMI group in the combined sex analysis was 0.39 (95\% confidence interval (CI): 0.04-0.73).

The childhood baseline of each group with and without the adult data was compared (Supplementary Table S1 online). Majority of the data were similar between the two groups. However, year of entrance and area of residence were different $(P<0.001$ and $P=0.001$, respectively) and the DBMI was marginal $(P=0.052)$. The results of the univariate and multivariate logistic regression analysis, in which the SUA cutoff level was $7 \mathrm{mg} / \mathrm{dl}$ using the same models described above, were not statistically significant (Supplementary Table S2 online).

\section{DISCUSSION}

The present study demonstrated that higher DBMI among primary school students is associated with increased SUA level in young adults compared with lower DBMI. The association between DBMI groups and young adult SUA levels was

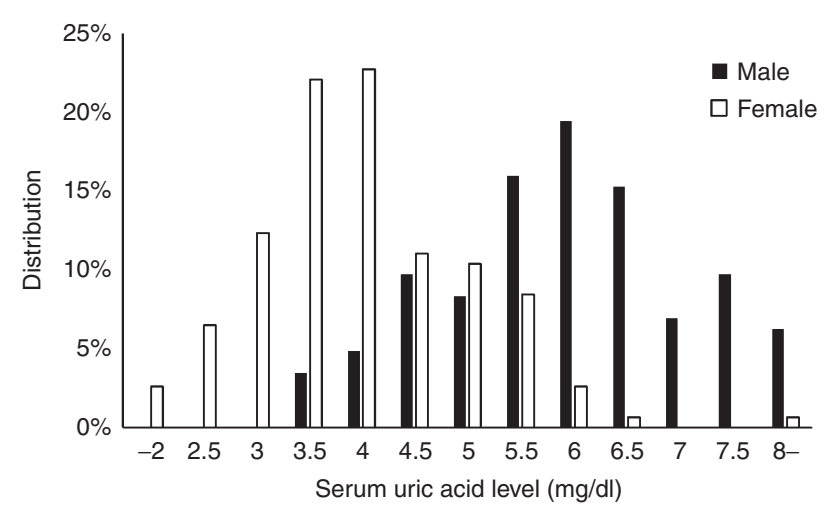

Figure 1. The distribution of SUA after reaching early adulthood ( $n=298$ ). Boys: black bar; Girls: white bar. 
Table 1. Comparison of physical growth and the associated factors during childhood in all subjects and for three groups of study participants with different degrees of BMI change between first and sixth grade and boys and girls as independent groups

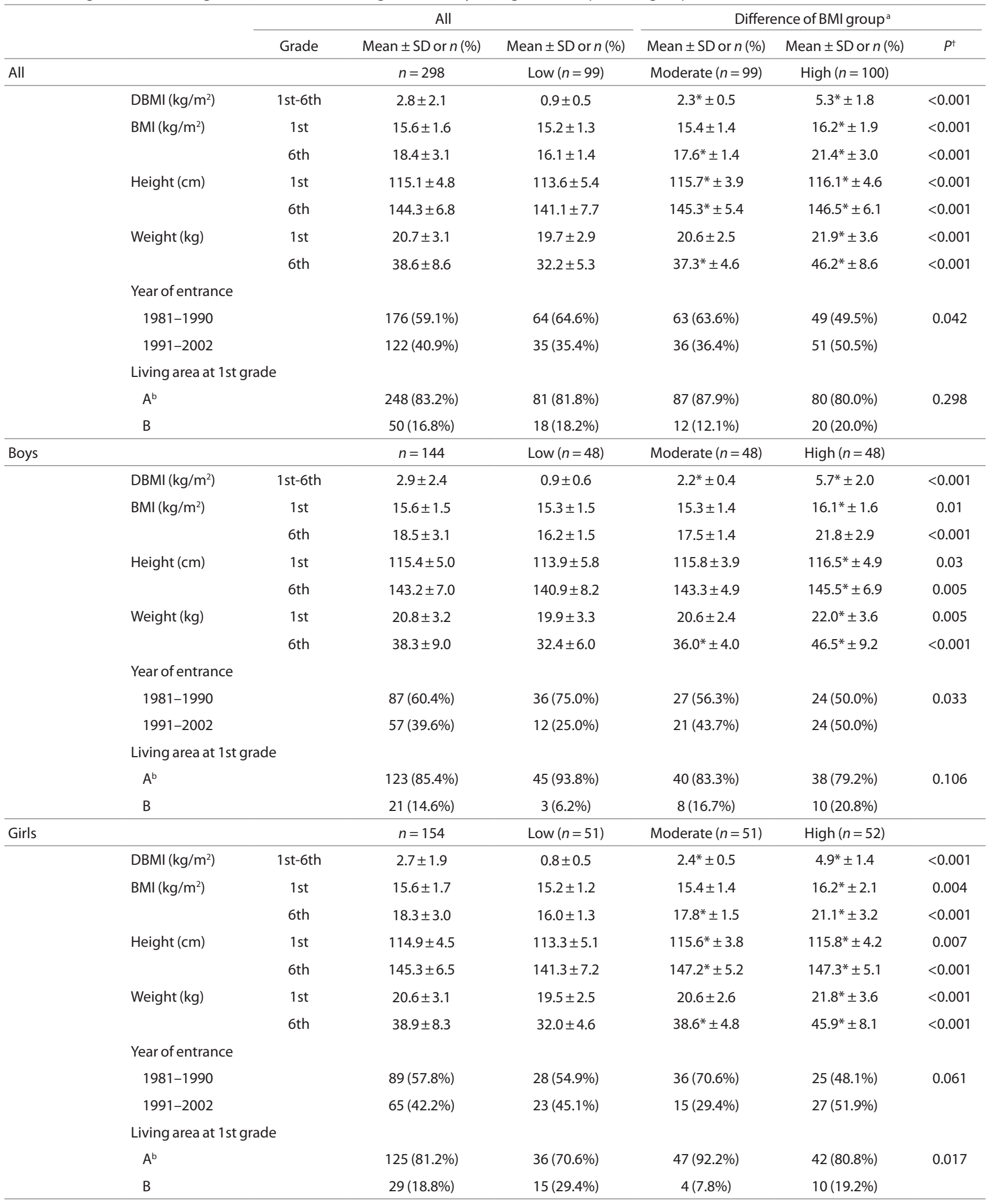

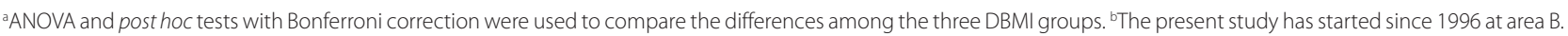

${ }^{*} P<0.05$ for post-hoc tests with Bonferroni correction using the low group as a reference. ${ }^{\dagger} P$ values for ANOVA, Pearson's chi-square test or Fisher's exact test. 


\section{Articles | Kuwaharaetal.}

Table 2. Comparison of physical condition, serum uric acid levels, and associated factors after reaching physical maturity in all study participants and for three groups with different degrees of BMI change between first and sixth grade for boys and girls as independent groups

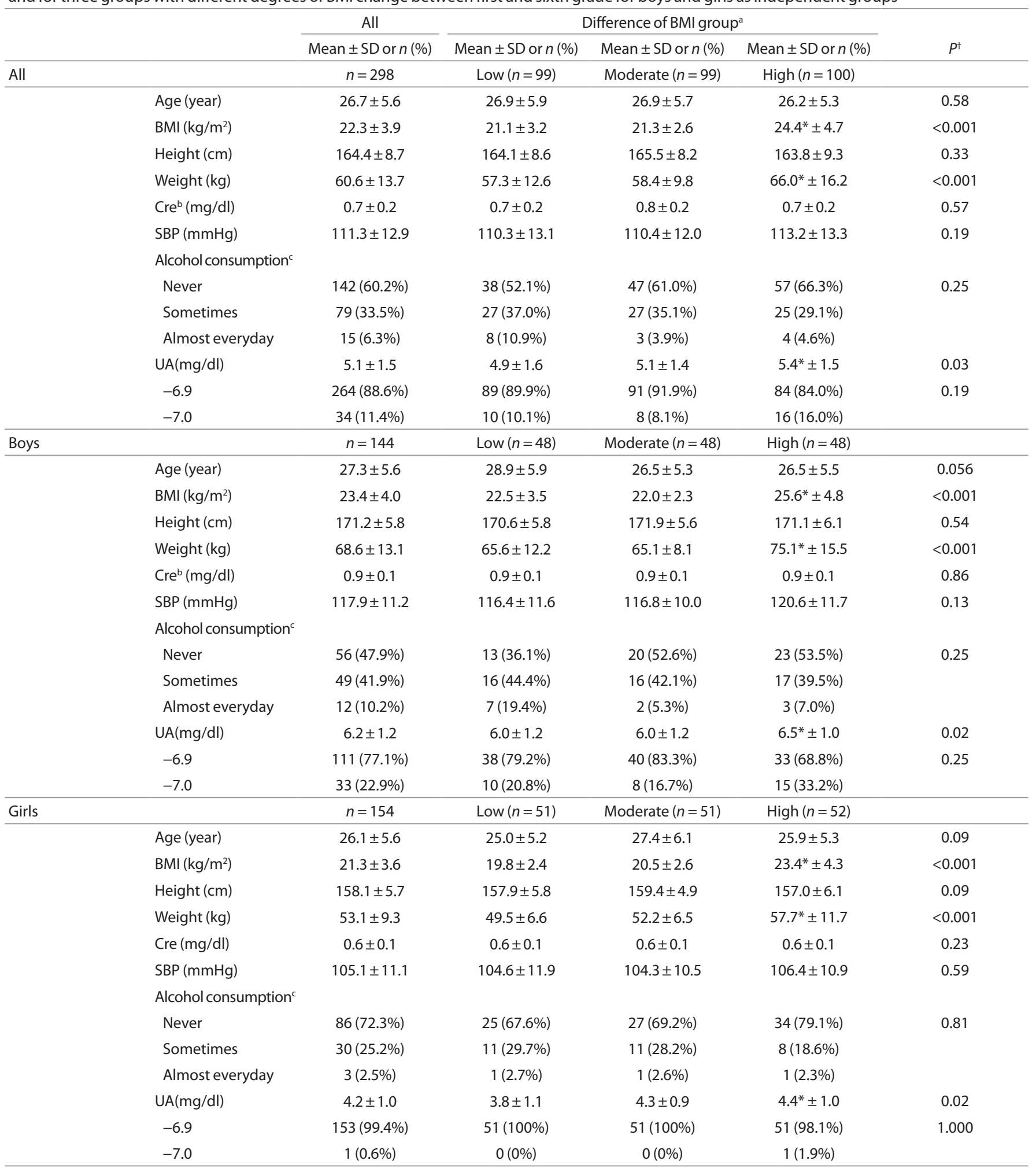

aANOVA and post-hoc tests with Bonferroni correction were used to compare the differences between the three DBMl groups. ${ }^{\mathrm{b} C} \mathrm{Creatinine}$ data was missing from one boy in the high BMI change group. 'Data were available for 117 boys and 119 girls.

SBP, systolic blood pressure.

"P $P 0.05$ for post-hoc tests with Bonferroni correction using the low group as a reference. ${ }^{\dagger} P$ values for ANOVA, Pearson's chi-square test or Fisher's exact test. 
Table 3. Regression analysis results of associations in differences in BMI between first and sixth grade with serum uric acid after reaching physical maturity

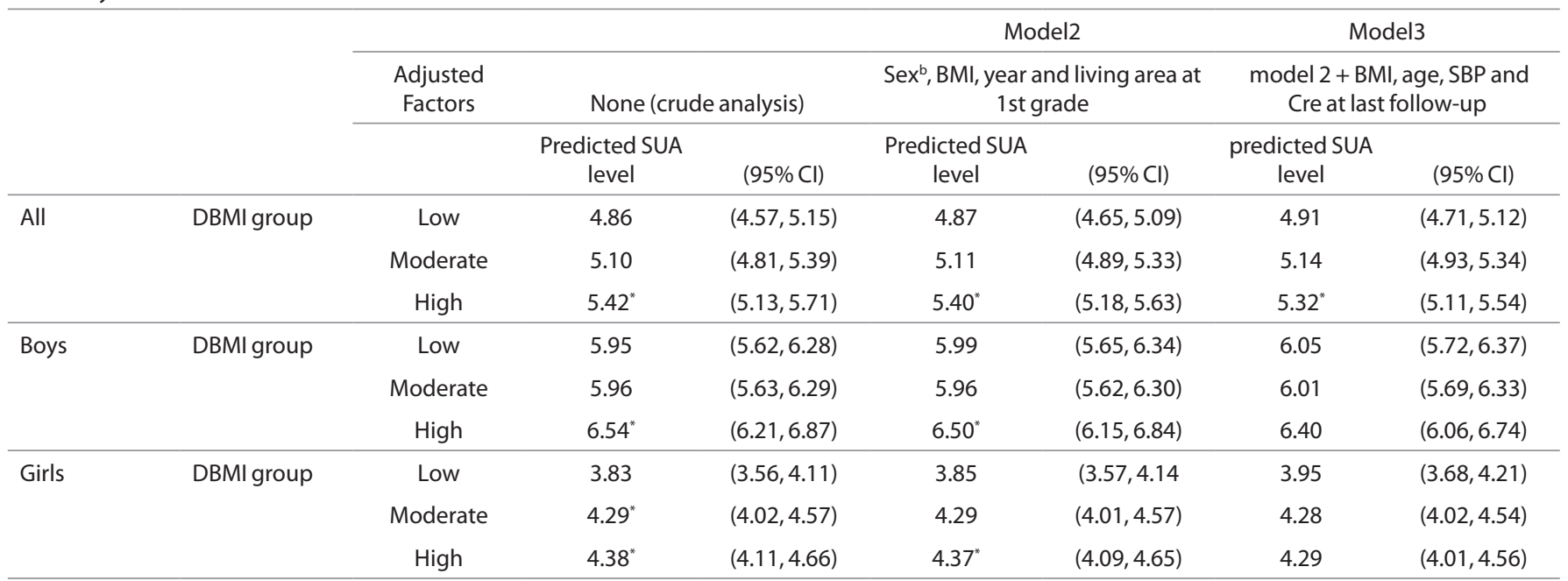

aNo creatinine data was available for one boy; therefore, data from 297 participants were analyzed in Model 3 . bSex was adjusted for only sex-combined analysis.

${ }^{*} P<0.05$ for univariate or multivariate regression analysis compared to the low group as a reference.

significant even after adjusting for baseline and adult BMIs. This association suggests that increases in BMI during childhood have an effect on SUA elevation, irrespective of the specific value of BMI.

The results of the sex-combined and sex-stratified analyses were similar, with a few exceptions. We did not observe a statistical interaction between sex and DBMI, and there was no difference in coefficients in the multivariate regression analysis between men and women. Small sample size may be one reason that some results of the sex-stratified adjusted multivariate regression analysis were not statistically significant.

It is possible that the study results are affected by residual confounders. SUA level is affected by alcohol consumption (23). Our results did not change after we adjusted for alcohol consumption but the number of subjects with data available for this analysis was relatively small. In addition, some medications affect SUA levels. Antihypertensive medications, such as calcium antagonists (24), angiotensin-converting enzyme inhibitors (25), and angiotensin II receptor blockers (26), decrease SUA level. Diuretics (25), $\beta$-blockers (27), and cyclosporine increase SUA level (28). However, since the subjects in our study were young, there may be few patients with serious diseases. There were only two hypertension patients and two diabetes patients receiving medication in our sample. When we conducted a multivariate regression analysis excluding the subjects who took medication for hypertension or diabetes, the results were not different (data not shown).

We found that SUA levels were associated with a BMI increase in childhood even after adjusting for BMI in adulthood. This suggests an SUA elevation pathway that is independent of adult obesity. One possibility is that dietary habits that result in excessive increases in BMI in childhood continue into adulthood, with a consequent increase in purine consumption.

The results of the multivariate logistic regression analysis did not show a statistically significant association between
DBMI tertile in childhood and hyperuricemia (SUA $\geq 7 \mathrm{mg}$ / dl) in adults. Perhaps a larger sample size would reveal a significant association. The mean SUA level among the male high DBMI group was $6.5 \pm 1.0 \mathrm{mg} / \mathrm{dl}$ in our study. A previous study demonstrated that even lower SUA levels were linearly associated with chronic kidney disease (29) and metabolic syndrome prevalence (30). Moreover, a cohort study concluded that higher SUA levels were an independent predictor of cardiovascular disease mortality, even for SUA $>6.27 \mathrm{mg} / \mathrm{dl}$ ( 373 $\mu \mathrm{mol} / \mathrm{l} / \mathrm{l}$ ) among males (31). Therefore, the SUA levels in our sample, particularly in the highest DBMI group for boys, could be a risk factor for cardiovascular disease in the future.

\section{Strengths and Limitations}

An important strength of this study is that it is one of the few investigations of childhood BMI and young adult SUA that use a longitudinal dataset. It is important to focus on childhood because early lifestyle trends often result in the development of a behavior that has a significant influence later in life. As the measurement protocol had been well standardized during the long study period, the dataset was reliable.

The study also has some limitations. First, the study sample may not be a representative of the entire Japan because the study areas included only a part of the Nagano Prefecture. Second, we did not have information regarding factors related to SUA, such as current diet and exercise patterns. Instead, we adjusted for adult BMI, Cre, and SBP to partly reflect the subjects' daily lives. Third, it is not evident whether DBMI between the first and sixth grades was representative of the change in BMI during childhood. Further studies are needed to determine the representative DBMI during childhood. Fourth, we could not determine the mechanism of SUA elevation among people who showed increased BMI during childhood in the present study. The elucidation of the mechanism for this association should be a topic for future research. 


\section{Articles | Kuwahara et al.}

We found that a large BMI increase in childhood was associated with elevated young adult SUA level after adjusting for BMI at baseline and in early adulthood. Additional researches on increases in BMI are required to clarify the mechanisms that may lead to later metabolic-related diseases.

\section{SUPPLEMENTARY MATERIAL}

Supplementary material is linked to the online version of the paper at http:// www.nature.com/pr

\section{ACKNOWLEDGMENTS}

We would like to express our gratitude to everyone involved in childhood health education in the areas studied. We also thank Yumi Mukoyama of Kashimanada Shinryojo, Ibaraki Prefecture for cooperation and support.

\section{AUTHOR CONTRIBUTIONS}

Study concept and design: E.K., Y.M., T.O., Y.N.; collection and assembly of data: H.K., A.N., F.M., H.U., Y.N.; statistical analysis: E.K., Y.M., Y.N.; manuscript write-up: E.K., Y.M., T.O., Y.N.; manuscript review and revision: E.K., Y.M., T.O., Y.N., A.N., H.U., Y.N., H.K., F.M.; final approval of the article: E.K., Y.M., T.O., Y.N., A.N., H.U., Y.N., H.K., F.M.

\section{STATEMENT OF FINANCIAL SUPPORT}

This work was supported by the Japan Society for the Promotion of the Science (JSPS) KAKENHI Grant-in-Aid for Young Scientists (B) Number 26860422.

Disclosure: The authors have no relevant financial relationships to disclose.

\section{REFERENCES}

1. Cannon PJ, Stason WB, Demartini FE, Sommers SC, Laragh JH. Hyperuricemia in primary and renal hypertension. N Engl J Med 1966;275: 457-64.

2. Barlow KA, Beilin LJ. Renal disease in primary gout. Q J Med 1968;37:79-96.

3. Gertler MM, Garn SM, Levine SA. Serum uric acid in relation to age and physique in health and in coronary heart disease. Ann Intern Med 1951;34:1421-31.

4. Obermayr RP, Temml C, Gutjahr G, Knechtelsdorfer M, Oberbauer R, Klauser-Braun R. Elevated uric acid increases the risk for kidney disease. J Am Soc Nephrol 2008;19:2407-13.

5. Sonoda H, Takase H, Dohi Y, Kimura G. Uric acid levels predict future development of chronic kidney disease. Am J Nephrol 2011;33:352-7.

6. Lehto S, Niskanen L, Rönnemaa T, Laakso M. Serum uric acid is a strong predictor of stroke in patients with non-insulin-dependent diabetes mellitus. Stroke 1998;29:635-9.

7. Moriarity JT, Folsom AR, Iribarren C, Nieto FJ, Rosamond WD. Serum uric acid and risk of coronary heart disease: Atherosclerosis Risk in Communities (ARIC) Study. Ann Epidemiol 2000;10:136-43.

8. Feig DI, Kang DH, Johnson RJ. Uric acid and cardiovascular risk. N Engl J Med 2008;359:1811-21.

9. Ishiro M, Takaya R, Mori Y, et al. Association of uric acid with obesity and endothelial dysfunction in children and early adolescents. Ann Nutr Metab 2013;62:169-76.

10. Ford ES, Li C, Cook S, Choi HK. Serum concentrations of uric acid and the metabolic syndrome among US children and adolescents. Circulation 2007;115:2526-32.

11. Barker DJ, Osmond C, Forsén TJ, Kajantie E, Eriksson JG. Trajectories of growth among children who have coronary events as adults. N Engl J Med 2005;353:1802-9.
12. Law CM, Shiell AW, Newsome CA, et al. Fetal, infant, and childhood growth and adult blood pressure: a longitudinal study from birth to 22 years of age. Circulation 2002;105:1088-92.

13. Ekelund U, Ong KK, Linné Y, et al. Association of weight gain in infancy and early childhood with metabolic risk in young adults. J Clin Endocrinol Metab 2007;92:98-103.

14. Ong KK, Ahmed ML, Emmett PM, Preece MA, Dunger DB. Association between postnatal catch-up growth and obesity in childhood: prospective cohort study. BMJ 2000;320:967-71.

15. Stettler N, Zemel BS, Kumanyika S, Stallings VA. Infant weight gain and childhood overweight status in a multicenter, cohort study. Pediatrics 2002;109:194-9.

16. Eriksson JG. Early growth and coronary heart disease and type 2 diabetes: findings from the Helsinki Birth Cohort Study (HBCS). Am J Clin Nutr 2011;94(6 Suppl):1799S-802S.

17. Eriksson JG, Forsén T, Tuomilehto J, Winter PD, Osmond C, Barker DJ. Catch-up growth in childhood and death from coronary heart disease: longitudinal study. BMJ 1999;318:427-31.

18. Kuwahara E, Asakura K, Nishiwaki Y, et al. Steeper increases in body mass index during childhood correlate with blood pressure elevation in adolescence: a long-term follow-up study in a Japanese community. Hypertens Res 2014;37:179-84.

19. Cole TJ. The LMS method for constructing normalized growth standards. Eur J Clin Nutr 1990;44:45-60.

20. Inokuchi M, Hasegawa T, Anzo M, Matsuo N. Standardized centile curves of body mass index for Japanese children and adolescents based on the 1978-1981 national survey data. Ann Hum Biol 2006;33:444-53.

21. Loeb JN. The influence of temperature on the solubility of monosodium urate. Arthritis Rheum 1972;15:189-92.

22. Mikkelsen WM, Dodge HJ, Valkenburg H. The distribution of serum uric acid values in a population unselected as to gout or hyperuricemia: Tecumseh, Michigan 1959-1960. Am J Med 1965;39:242-51.

23. Statland BE, Winkel P, Bokelund H. Factors contributing to intra-individual variation of serum constituents. 2. Effects of exercise and diet on variation of serum constituents in healthy subjects. Clin Chem 1973;19:1380-3.

24. Miyazaki S, Hamada T, Hirata S, et al. Effects of azelnidipine on uric acid metabolism in patients with essential hypertension. Clin Exp Hypertens 2014;36:447-53.

25. Reyes AJ. Cardiovascular drugs and serum uric acid. Cardiovasc Drugs Ther 2003;17:397-414.

26. Iwanaga T, Sato M, Maeda T, Ogihara T, Tamai I. Concentration-dependent mode of interaction of angiotensin II receptor blockers with uric acid transporter. J Pharmacol Exp Ther 2007;320:211-7.

27. Høieggen A, Alderman MH, Kjeldsen SE, et al.; LIFE Study Group. The impact of serum uric acid on cardiovascular outcomes in the LIFE study. Kidney Int 2004;65:1041-9.

28. Lin HY, Rocher LL, McQuillan MA, Schmaltz S, Palella TD, Fox IH. Cyclosporine-induced hyperuricemia and gout. N Engl J Med 1989;321:287-92.

29. Chang HY, Tung CW, Lee PH, et al. Hyperuricemia as an independent risk factor of chronic kidney disease in middle-aged and elderly population. Am J Med Sci 2010;339:509-15.

30. Choi HK, Ford ES. Prevalence of the metabolic syndrome in individuals with hyperuricemia. Am J Med 2007;120:442-7.

31. Liese AD, Hense HW, Löwel H, Döring A, Tietze M, Keil U. Association of serum uric acid with all-cause and cardiovascular disease mortality and incident myocardial infarction in the MONICA Augsburg cohort. World Health Organization Monitoring Trends and Determinants in Cardiovascular Diseases. Epidemiology 1999;10:391-7. 